\title{
The effect of physical multimorbidity, mental health conditions and socioeconomic deprivation on unplanned admissions to hospital: a retrospective cohort study
}

\author{
Rupert A. Payne MBChB PhD, Gary A. Abel PhD, Bruce Guthrie MBBChir PhD, Stewart W. Mercer MBChB PhD
}

\begin{abstract}
Background: Multimorbidity, the presence of more than 1 long-term disorder, is associated with increased use of health services, but unplanned admissions to hospital may often be undesirable. Furthermore, socioeconomic deprivation and mental health comorbidity may lead to additional unplanned admissions. We examined the association between unplanned admission to hospital and physical multimorbidity, mental health and socioeconomic deprivation.
\end{abstract}

Methods: We conducted a retrospective cohort study using data from 180815 patients aged 20 years and older who were registered with 40 general practices in Scotland. Details of 32 physical and 8 mental health morbidities were extracted from the patients' electronic health records (as of Apr. 1, 2006) and linked to hospital admission data. We then recorded the occurrence of unplanned or potentially preventable unplanned acute (nonpsychiatric) admissions to hospital in the subsequent 12 months. We used logistic regression models, adjusting for age and sex, to determine associations between unplanned or potentially preventable unplanned admissions to hospital and physical multimorbidity, mental health and socioeconomic deprivation.
Results: We identified 10828 (6.0\%) patients who had at least 1 unplanned admission to hospital and 2037 (1.1\%) patients who had at least 1 potentially preventable unplanned admission to hospital. Both unplanned and potentially preventable unplanned admissions were independently associated with increasing physical multimorbidity (for $\geq 4 \mathrm{v}$. 0 conditions, odds ratio [OR] 5.87 [95\% confidence interval (CI) 5.45-6.32] for unplanned admissions, OR 14.38 [95\% Cl 11.87-17.43] for potentially preventable unplanned admissions), mental health conditions (for $\geq 1$ v. 0 conditions, OR 2.01 [95\% Cl 1.92-2.09] for unplanned admissions, OR 1.80 [95\% Cl 1.641.97] for potentially preventable unplanned admissions) and socioeconomic deprivation (for most v. least deprived quintile, OR 1.56 [95\% Cl 1.43-1.70] for unplanned admissions, OR 1.98 [95\% Cl 1.63-2.41] for potentially preventable unplanned admissions).

Interpretation: Physical multimorbidity was strongly associated with unplanned admission to hospital, including admissions that were potentially preventable. The risk of admission to hospital was exacerbated by the coexistence of mental health conditions and socioeconomic deprivation.
$\mathrm{M}$ ultimorbidity - usually defined as the presence of more than 1 longterm disorder - is becoming the norm rather than the exception as populations age. ${ }^{1,2}$ A recent study found that most people older than 65 years of age had multimorbidity, and the mean number of comorbidities per person increased with age; ${ }^{1}$ however, multimorbidity is not confined to older adults. ${ }^{3}$

Multimorbidity is associated with a range of adverse outcomes. People with multimorbidity have worse physical, social and psychological quality of life ${ }^{4}$ and increased mortality. ${ }^{5}$ Mental health conditions often accompany and exacerbate long-term physical conditions, leading to poor health outcomes, reduced quality of life and increased costs. ${ }^{1,6,7}$ Furthermore, health services are largely organized to provide care for single diseases, particularly in hospitals or under specialist care. Indeed, many aspects of care are poor for patients with multimorbidity. ${ }^{8-10}$ This situation may be further aggravated among patients who are socioeconomically disadvantaged, because they often have poorer health and higher health care needs, while also experiencing poorer provision of services, than their more advantaged counterparts. ${ }^{11}$ A lack of social and personal resources, coupled with multiple stresses, makes coping difficult for these patients, ${ }^{12}$ and the multiplicity of physical, psychological and social
Competing interests: None declared.

This article has been peer reviewed.

Correspondence to:

Rupert A. Payne,

rap55@medschl.cam.ac.uk

CMAJ 2013. DOI:10.1503 /cmaj.121349 
problems means that family physicians sometimes struggle to support patients with multimorbidity in deprived settings. ${ }^{13}$

Multimorbidity is associated with increased use of health services; however, whereas high use of primary and specialist ambulatory care may be seen as an appropriate response to multimorbidity, frequent unplanned admissions to hospital will often be undesirable. ${ }^{14}$ Unfortunately, there are relatively few large studies that have examined the association between multimorbidity and unplanned hospital admissions. ${ }^{15-17}$ Moreover, such studies did not separately examine physical and mental health morbidity and did not account for the additional effect of socioeconomic deprivation - shortcomings we hope to have addressed. Using linked routine clinical primary care and hospital data, we sought to determine the association between unplanned admissions to hospital and physical multimorbidity, as well as any additional effect of mental health morbidity and socioeconomic deprivation.

\section{Methods}

\section{Study design}

We used a retrospective cohort study design using data from routine primary care electronic medical records linked to national administrative data on acute hospital admissions. We included data from the records of all patients aged 20 years and older on Apr. 1, 2006, and we followed patients for 12 months to identify admissions to hospital. The study was approved by the National Health Service (NHS) National Services Scotland Privacy Advisory Committee.

\section{Data sources}

Forty general practices contributing data on all permanently registered patients to the Scottish Practice Team Information dataset participated in the study. This national dataset compiles information on clinical activity and morbidity, and is broadly representative of the Scottish population. ${ }^{18}$ We extracted complete copies of all coded data (e.g., demographics, prescribing, diagnoses) from each participating practice. The Information Services Division of the NHS National Services Scotland used probabilistic matching to link clinical data to acute hospital admissions data (the Scottish Morbidity Record [SMR-01]). These records are generated for all medical discharges and transfers from acute care hospitals, excluding visits to emergency departments and admissions to maternity and psychiatric units. Admissions may be classified as routine, urgent or emergency. For the period 2004-2006, SMR01 data have been shown to be $99 \%$ complete, with $88 \%$ accuracy for admission diagnosis (compared with clinical case records) based on the 3-digit codes of the International Statistical Classification of Diseases and Related Health Problems, 10th revision. ${ }^{19}$

\section{Multimorbidity}

Using methods and condition definitions that have been previously defined, ${ }^{1}$ we searched the linked data for the presence of 40 conditions (Appendix 1, available at www.cmaj.ca/lookup /suppl/doi:10.1503/cmaj.121349/-/DC1) on Apr. 1, 2006. We used a simple unweighted count of physical health conditions $(0,1,2,3$, or $\geq 4$ ) to quantify physical multimorbidity. We included the presence of any of 8 mental health conditions as a binary variable $(\geq 1$ or 0$)$.

\section{Deprivation}

We quantified socioeconomic deprivation using the national Scottish Index of Multiple Deprivation. ${ }^{20}$ This measure is based on small geographic areas and encompasses a range of domains, such as education, employment, services and crime, to address the multidimensional nature of deprivation. The index is widely used to facilitate targeting of policies and resources to mitigate socioeconomic inequalities.

\section{Outcomes}

We identified people with at least 1 urgent or emergency admission (nonpsychiatric) to an acute care hospital (henceforth referred to as unplanned admissions), or potentially preventable urgent or emergency admission to hospital (henceforth referred to as potentially preventable unplanned admissions) during the 12-month period starting Apr. 1, 2006, and as recorded in the SMR-01. Potentially preventable admissions are those for conditions that are considered the most preventable by better primary and outpatient care, defined using a standard NHS Scotland list (Appendix 2, available at www.cmaj.ca/lookup /suppl/doi:10.1503/cmaj.121349/-/DC1). ${ }^{21}$

\section{Statistical analysis}

We calculated unadjusted admission rates for the whole population, and by sex, age group, Scottish Index of Multiple Deprivation quintile (in which quintile 1 is the least deprived), physical multimorbidity count and the presence of a mental health condition. We constructed fixed-effect univariable and mixed-effect multivariable logistic regression models with hospital admission (unplanned or potentially preventable unplanned) as the outcome variable. Sex, age, deprivation quintile, physical multimorbidity count and mental health were included as fixed covariates. Individual practices 
may vary in their underlying tendency to admit patients, which may confound the associations of interest. Thus, we incorporated a random effect for practice in our multivariable models to account for the clustering of patients within practices. We then calculated predicted probabilities for admission to hospital from these models.

We fitted additional models examining plausible interactions between physical multimorbidity and mental health, and deprivation, age and sex. We also conducted a number of sensitivity analyses to examine whether the manner in which conditions were counted or types of conditions might influence the effect of deprivation, physical multimorbidity or mental health (Appendix 3, available at www.cmaj.ca/lookup/suppl /doi:10.1503/cmaj.121349/-/DC1).

\section{Results}

Our analysis included data from 180815 patients (Table 1). The median age of patients was 49 (interquartile range 36-63) years (data not shown), and $89076(49.3 \%)$ were men (Table 1). The prevalence of physical multimorbidity and mental health morbidity increased with deprivation (Appendices 4 and 5, available at www.cmaj.ca /lookup/suppl/doi:10.1503/cmaj.121349/-/DC1).

During the 12-month follow-up period, 10828 $(6.0 \%)$ patients had at least 1 unplanned admission to hospital, and 2037 (1.1\%) patients had at least 1 potentially preventable unplanned admission to hospital (Table 1). Of the 17858 patients with 4 or more physical health conditions, 3659 (20.5\%) had unplanned admissions to hospital,

Table 1: Charactertistics of patients included in the study and unadjusted rates of admission to hospital by characteristic

\begin{tabular}{|c|c|c|c|}
\hline \multirow{3}{*}{$\begin{array}{l}\text { Characteristic } \\
\text { All }\end{array}$} & \multirow{3}{*}{$\begin{array}{r}\text { Total } \\
180815\end{array}$} & \multicolumn{2}{|c|}{ No. $(\%) *$ of patients } \\
\hline & & $\begin{array}{l}\text { At least } 1 \text { unplanned } \\
\text { admission }\end{array}$ & $\begin{array}{c}\text { At least } 1 \text { potentially } \\
\text { preventable unplanned } \\
\text { admission }\end{array}$ \\
\hline & & $10828 \quad(6.0)$ & 2037 (1.1) \\
\hline \multicolumn{4}{|l|}{ Sex } \\
\hline Male & 89076 & 5209 & $984(1.1)$ \\
\hline Female & 91739 & $5619 \quad(6.1)$ & $1053(1.1)$ \\
\hline \multicolumn{4}{|l|}{ Age, yr } \\
\hline $20-34$ & 38500 & 1237 & $170(0.4)$ \\
\hline $35-44$ & 35934 & 1180 & $158(0.4)$ \\
\hline $45-54$ & 34227 & 1310 & $195(0.6)$ \\
\hline $55-64$ & 30827 & $1679 \quad(5.4)$ & $316(1.0)$ \\
\hline $65-74$ & 22053 & 1910 & $426(1.9)$ \\
\hline$\geq 75$ & 19274 & $3512(18.2)$ & $772(4.0)$ \\
\hline \multicolumn{4}{|l|}{ Deprivation quintile } \\
\hline 1 (least deprived) & 31121 & 1281 & $184(0.6)$ \\
\hline 2 & 37261 & $1949 \quad(5.2)$ & $348(0.9)$ \\
\hline 3 & 45823 & $2685 \quad(5.9)$ & $529(1.2)$ \\
\hline 4 & 36098 & $2424 \quad(6.7)$ & 469 (1.3) \\
\hline 5 (most deprived) & 30512 & $2489 \quad(8.2)$ & $507(1.7)$ \\
\hline \multicolumn{4}{|c|}{ No. of physical conditions } \\
\hline 0 & 83045 & 1971 & $195(0.2)$ \\
\hline 1 & 42825 & 1872 & $268(0.6)$ \\
\hline 2 & 23344 & 1791 & $322(1.4)$ \\
\hline 3 & 13743 & 1535 (11.2) & $300 \quad(2.2)$ \\
\hline$\geq 4$ & 17858 & 3659 (20.5) & $952(5.3)$ \\
\hline \multicolumn{4}{|l|}{$\begin{array}{l}\text { No. of mental health } \\
\text { conditions }\end{array}$} \\
\hline 0 & 137876 & 6185 & $1122(0.8)$ \\
\hline$\geq 1$ & 42939 & $4643(10.8)$ & 915 (2.1) \\
\hline
\end{tabular}


compared with $2.4 \%$ of patients (1971/83 045) with no physical health conditions; the corresponding rates for preventable unplanned hospital admissions were $5.3 \%$ of patients with 4 or more comorbidities (952/17 858) and $0.2 \%$ of patients with no comorbidities (195/83 045) (Table 1).

Patients with a mental health disorder were more likely than patients with no mental health disorder to have unplanned $(10.8 \%$ [4653/42 939] v. $4.5 \%$ [6185/137 876]) or potentially preventable unplanned admissions (2.1\% [915/42 939] v. $0.8 \%$ [1122/137 876]) (Table 1). People in the most socioeconomically deprived quintile were more likely than those in the least deprived quintile to have unplanned $(8.2 \%$ [2489/30 512] v. $4.1 \%$ [1281/31 121]) or potentially preventable unplanned $(1.7 \%$ [507/30 512] v. $0.6 \%$ [184/31 121]) admissions to hospital.

Only $9.9 \%$ of patients (17 858/180 815) had 4 or more comorbid physical conditions, but these patients accounted for $33.8 \%$ (3659/10 828) of patients with unplanned admissions to hospital and $46.7 \%$ (952/2037) of patients with potentially preventable unplanned admissions to hospital. Simi- larly, although only $23.7 \%$ (42 939/180 815) of patients had a mental health condition, they accounted for $42.9 \%$ (4643/10 828) of patients with an unplanned admission to hospital and $44.9 \%$ $(915 / 2037)$ of patients with a potentially preventable unplanned admission to hospital. Furthermore, $23.0 \%$ (2489/10 828) of all patients with an unplanned admission to hospital were in the most socioeconomically deprived quintile, compared with 11.8\% (1281/10 828) in the least deprived.

Increasing age and male sex were associated with increased risk for both types of admission to hospital, although smaller increases were also seen in the youngest age group (Tables 2 and 3). Compared with having no physical health conditions, having 4 or more physical health conditions was associated with higher risks of unplanned admissions (adjusted odds ratio [OR] $5.87,95 \%$ confidence interval [CI] 5.45-6.32) and potentially preventable unplanned admissions (adjusted OR 14.38, 95\% CI 11.87-17.43) (Tables 2 and 3). The presence of a mental health condition was also associated with an increased risk of unplanned admission (adjusted OR 2.01,

Table 2: Logistic regression models for unplanned admissions to hospital

\begin{tabular}{|c|c|c|c|c|}
\hline Covariate & Unadjusted OR (95\% CI) & $p$ value* & Adjusted OR† (95\% Cl) & $p$ value* \\
\hline Male sex & $0.95 \quad(0.92-0.99)$ & 0.01 & $1.22(1.17-1.27)$ & $<0.001$ \\
\hline Age, yr & & $<0.001$ & & $<0.001$ \\
\hline $20-34$ & $0.83 \quad(0.77-0.90)$ & & $1.15(1.05-1.24)$ & \\
\hline $35-44$ & $0.85 \quad(0.79-0.92)$ & & $1.03(0.95-1.12)$ & \\
\hline $65-74$ & $2.38 \quad(2.22-2.56)$ & & $1.41(1.30-1.52)$ & \\
\hline$\geq 75$ & $5.60 \quad(5.24-5.98)$ & & $2.59(2.40-2.79)$ & \\
\hline Deprivation quintile & & $<0.001$ & & $<0.001$ \\
\hline 1 (least deprived) & 1.00 (ref) & & 1.00 (ref) & \\
\hline 5 (most deprived) & $2.07 \quad(1.93-2.22)$ & & $1.56(1.43-1.70)$ & \\
\hline No. of physical conditions & & $<0.001$ & & $<0.001$ \\
\hline 0 & 1.00 (ref) & & 1.00 (ref) & \\
\hline 1 & $1.88 \quad(1.76-2.01)$ & & $1.70(1.59-1.82)$ & \\
\hline 2 & $3.42 \quad(3.20-3.65)$ & & $2.69(2.50-2.89)$ & \\
\hline 3 & $5.17 \quad(4.83-5.54)$ & & $3.47(3.21-3.76)$ & \\
\hline$\geq 4$ & $10.60(10.01-11.23)$ & & $5.87(5.45-6.32)$ & \\
\hline Mental health condition & $2.58 \quad(2.48-2.69)$ & $<0.001$ & $2.01(1.92-2.09)$ & $<0.001$ \\
\hline
\end{tabular}


95\% CI 1.92-2.09) and potentially preventable unplanned admission (OR 1.80, 95\% CI 1.641.97). In addition, socioeconomic deprivation was independently associated with unplanned admission to hospital (OR 1.56, 95\% CI 1.43$1.70)$ and potentially preventable unplanned admission to hospital (OR 1.98, 95\% CI 1.632.41) (Tables 2 and 3). The random effect for practice provided strong evidence for modest variation in rates of admission between practices (joint test $p<0.001$; Tables 2 and 3 ).

Although we saw statistically significant interactions between physical multimorbidity and mental health, and deprivation, age and sex, absolute differences were small; including the interactions did not make any substantive difference to our interpretation. In the interest of clarity, we therefore only present the more parsimonious model without interactions.

The results of our sensitivity analyses support the findings of our main analysis (Appendix 3). In particular, the effect of deprivation persisted independently of the manner in which morbidity was quantified, although confounding by other unmeasured health factors such as disease sever- ity cannot be discounted. Using alternative physical condition counts resulted in little change in the effect size for physical multimorbidity. Different mental health factors appeared to have similar effect sizes, although there was evidence that alcohol misuse conveyed greater risk, as did the presence of a mental health condition.

We saw a strong association between unplanned admission to hospital and number of physical health conditions (Figure 1) and, to a lesser degree, between the presence of mental health conditions, socioeconomic deprivation and unplanned admissions.

The synergistic effect of physical multimorbidity, mental health and deprivation was notably large. Compared with people living in the least deprived areas with no mental or physical health conditions, people in the most socioeconomically deprived areas who had a mental health condition and 4 or more physical health conditions had about 18 times the odds of an unplanned admission to hospital (OR 18.34, 95\% CI 16.40-20.52) and about 51 times the odds of a potentially preventable admission to hospital (OR 51.20, 95\% CI 39.06-67.11).

Table 3: Logistic regression models for potentially preventable unplanned admissions to hospital

\begin{tabular}{|c|c|c|c|c|}
\hline Covariate & $\begin{array}{l}\text { UnadjustedOR } \\
(95 \% \mathrm{Cl})\end{array}$ & $p$ value* & $\begin{array}{l}\text { Adjusted OR }+ \\
\quad(95 \% \mathrm{Cl})\end{array}$ & $p$ value* \\
\hline Male sex & $0.96 \quad(0.88-1.05)$ & 0.4 & $1.26 \quad(1.15-1.38)$ & $<0.001$ \\
\hline $20-34$ & $0.77 \quad(0.63-0.95)$ & & $1.34 \quad(1.09-1.67)$ & \\
\hline $35-44$ & $0.77 \quad(0.62-0.95)$ & & $1.07 \quad(0.87-1.33)$ & \\
\hline $55-64$ & $1.81 \quad(1.51-2.16)$ & & $1.22 \quad(1.01-1.46)$ & \\
\hline $65-74$ & $3.44 \quad(2.90-4.08)$ & & $1.56 \quad(1.30-1.87)$ & \\
\hline$\geq 75$ & $7.28 \quad(6.22-8.53)$ & & $2.39 \quad(2.01-2.84)$ & \\
\hline Deprivation quintile & & $<0.001$ & & $<0.001$ \\
\hline 1 (least deprived) & 1.00 (ref) & & 1.00 (ref) & \\
\hline 5 (most deprived) & $2.84 \quad(2.40-3.37)$ & & $1.98 \quad(1.63-2.41)$ & \\
\hline No. of physical conditions & & $<0.001$ & & $<0.001$ \\
\hline 0 & 1.00 (ref) & & 1.00 (ref) & \\
\hline 1 & $2.68 \quad(2.22-3.22)$ & & $2.50 \quad(2.07-3.03)$ & \\
\hline 2 & $5.94 \quad(4.97-7.10)$ & & $4.93 \quad(4.06-5.99)$ & \\
\hline 3 & $9.48 \quad(7.91-11.37)$ & & $6.82 \quad(5.55-8.37)$ & \\
\hline$\geq 4$ & $23.93(20.49-27.93)$ & & 14.38 (11.87-17.43) & \\
\hline Mental health condition & $2.65 \quad(2.43-2.90)$ & $<0.001$ & $1.80 \quad(1.64-1.97)$ & $<0.001$ \\
\hline \multicolumn{5}{|c|}{$\begin{array}{l}\text { Note: } \mathrm{Cl}=\text { confidence inteval, } \mathrm{OR}=\text { odds ratio, } \mathrm{ref}=\text { reference. } \\
{ }^{*} p \text { values based on joint tests, which test the overall differences between the individual categories of a given variable. } \\
\text { tAdjusted for other variables listed in the table. The adjusted model includes a random effect for practice. Intraclass correlation coefficient for practice variation } \\
0.019 \text {. OR for } 95 \% \text { midrange of practice variation (i.e., } 2.5 \text { and } 97.5 \text { percentiles, compared with average practice), } 0.61-1.63 \text {. }\end{array}$} \\
\hline
\end{tabular}




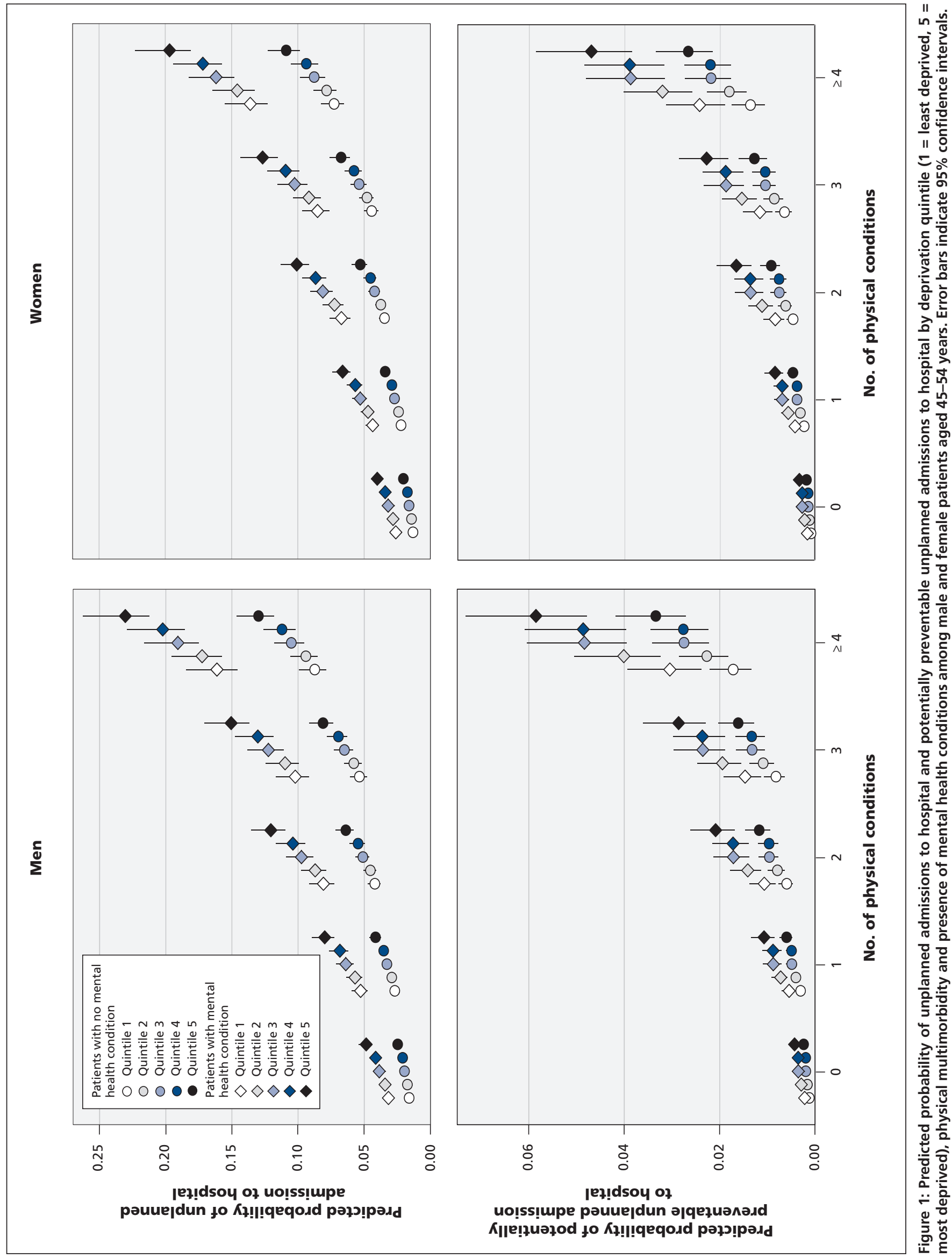




\section{Interpretation}

People with multimorbidity were more likely than people without multimorbidity to have unplanned admissions to hospital. Associations with age were reduced but not eliminated by accounting for multimorbidity. The residual association was likely due to frailty and reduced functional status not fully captured by a morbidity count. The effect of physical multimorbidity on admission to hospital was similarly attenuated after adjusting for other factors but remained substantial. This effect was particularly true for potentially preventable unplanned admissions, where patients with 4 or more physical conditions had a predicted probability of admission about 14 times that of people with no physical health conditions. Even after accounting for physical multimorbidity, the presence of mental health conditions and greater socioeconomic deprivation were associated with higher rates of admission. The people most likely to be admitted were those with mental illness in addition to high levels of physical multimorbidity and living in deprived areas.

Just under $10 \%$ of patients had 4 or more physical health conditions, yet these patients accounted for one-third of unplanned and almost one-half of potentially preventable unplanned admissions to hospital. Reducing unplanned admissions therefore requires interventions to improve care for people with multimorbidity. This may necessitate better, coordinated generalist care in the community from family physicians, ${ }^{22}$ particularly in countries with welldeveloped primary care systems. In addition, it could require improved in-patient generalist care from geriatricians ${ }^{23}$ or other hospitalists. ${ }^{24}$ Such improvements are especially important for patients with mental health morbidity, among whom we saw increased admissions to acute, nonpsychiatric hospitals (overwhelmingly for physical problems). However, the chasm between specialist physical and mental health services needs to be bridged; simply increasing the role of geriatricians or hospitalists may not adequately address this problem.

Even after accounting for physical multimorbidity and mental health conditions, both of which are more common and occur at younger ages among people in socioeconomically deprived areas, ${ }^{1}$ the people who are most socioeconomically disadvantaged were still more likely to have an unplanned admission to hospital. This trend may be due to unmeasured social problems creating psychological distress, as well as to fewer personal and community resources being available to these patients. ${ }^{13}$ Better models of care should thus be developed in the most deprived areas. These issues may be particularly relevant for potentially preventable admissions, which by definition are more likely to respond to community-based interventions.

Few large studies have examined the associations between multimorbidity and unplanned admission to hospital. In a large US population of people accessing Medicare, Wolff and colleagues found an OR of 99 for potentially preventable admissions among patients with more than 3 physical health conditions compared with patients with no physical health conditions. ${ }^{17}$ However, no adjustment was made for socioeconomic status. In another US study, the percentage of patients with more than 4 conditions was $50 \%$ higher among patients with hospital readmissions than among those with no readmissions. ${ }^{25}$ Reid and colleagues found high users of Canadian health services to be from more deprived areas and to have more multimorbidity, with $5 \%$ of users consuming $30 \%$ of resources. ${ }^{15}$ More recently, Salisbury and colleagues described similar findings in relation to general practice consultations in the United Kingdom. ${ }^{10}$ A smaller Irish study showed a positive association for both multimorbidity and deprivation with various aspects of health service usage, including an OR of 4.5 for admission among patients with more than 4 conditions. ${ }^{16}$ Our results are broadly consistent with those of previous studies and provide additional information about mental health and socioeconomic deprivation. Differences may be explained by variation in health care systems, in the nature and baseline risks of the populations studied and in the definitions of multimorbidity and outcomes.

\section{Strengths and limitations}

We used routine data that were linked, which enabled us to reliably identify hospital admissions. In addition, our study population was large. However, routine data are collected for clinical and administrative purposes and are thus likely to have at least some associated inaccuracies, although both of the datasets we used have been shown to be high quality. ${ }^{1926}$

We determined deprivation using an areabased measure. Although such measures are widely used by researchers and policy-makers, they are subject to some misclassification at the individual level. In addition, small pockets of extreme deprivation in Scotland are not captured by this dataset. However, these issues will likely have underestimated the effect of deprivation.

We used a simple count of multimorbidity and a dichotomous variable for mental health, and we were unable to account for severity or social complexity. However, there is a necessary 
trade-off between larger studies with routine data such as ours, and smaller studies employing specially collected detailed information, which may lack power to examine important questions. Furthermore, alternative methods of quantifying multimorbidity, as per our sensitivity analyses, support the simpler models' findings.

Finally, we did not account for previous admissions to hospital, a well-recognized predictor of subsequent admission. However, our aim was to examine the adverse consequences of multimorbidity, rather than constructing an admission-prediction model. Including previous admissions - themselves a likely consequence of pre-existing clinical conditions - would likely underestimate the actual effect of multimorbidity.

\section{Conclusion}

We provide strong evidence that physical multimorbidity substantially affects the use of acute hospital services, including admissions considered potentially preventable through optimal management of primary care. This effect was exacerbated by the coexistence of mental illness and socioeconomic deprivation. Further research is needed to examine whether primary care interventions targeting multimorbidity, particularly for patients in the most socioeconomically deprived areas, can improve quality of life and reduce hospital use.

\section{References}

1. Barnett K, Mercer SW, Norbury M, et al. Epidemiology of multimorbidity and implications for health care, research, and medical education: a cross-sectional study. Lancet 2012;380:37-43.

2. Fortin M, Bravo G, Hudon C, et al. Prevalence of multimorbidity among adults seen in family practice. Ann Fam Med 2005;3:223-8.

3. Taylor AW, Price K, Gill TK, et al. Multimorbidity — not just an older person's issue. Results from an Australian biomedical study. BMC Public Health 2010;10:718.

4. Fortin M, Lapointe L, Hudon C, et al. Multimorbidity and quality of life in primary care: a systematic review. Health Qual Life Outcomes 2004;2:51.

5. Perkins AJ, Kroenke K, Unutzer J, et al. Common comorbidity scales were similar in their ability to predict health care costs and mortality. J Clin Epidemiol 2004;57:1040-8.

6. Gunn JM, Ayton DR, Densley K, et al. The association between chronic illness, multimorbidity and depressive symptoms in an Australian primary care cohort. Soc Psychiatry Psychiatr Epidemiol 2012;47:175-84.

7. Naylor C, Parsonage M, McDaid D, et al. Long-term conditions and mental health: the cost of co-morbidities. London (UK): The King's Fund and Centre for Mental Health; 2012.

8. Fung CH, Setodji CM, Kung FY, et al. The relationship between multimorbidity and patients' ratings of communication. J Gen Intern Med 2008;23:788-93.

9. The Commonwealth Fund. 2010 Commonwealth Fund International Health Policy Survey. New York (NY): The Fund; 2010.

10. Salisbury C, Johnson L, Purdy S, et al. Epidemiology and impact of multimorbidity in primary care: a retrospective cohort study. Br J Gen Pract 2011;61:e12-21.

11. Mercer SW, Watt GC. The inverse care law: clinical primary care encounters in deprived and affluent areas of Scotland. Ann Fam Med 2007;5:503-10.
12. Bayliss EA, Steiner JF, Fernald DH, et al. Descriptions of barriers to self-care by persons with comorbid chronic diseases. Ann Fam Med 2003;1:15-21.

13. O'Brien R, Wyke S, Guthrie B, et al. An "endless struggle": a qualitative study of general practitioners' and practice nurses' experiences of managing multimorbidity in socio-economically deprived areas of Scotland. Chronic Illn 2011;7:45-59.

14. Purdy S. Avoiding hospital admissions: What does the research evidence say? London (UK): The King's Fund; 2010.

15. Reid R, Evans R, Barer M, et al. Conspicuous consumption: characterizing high users of physician services in one Canadian province. J Health Serv Res Policy 2003;8:215-24.

16. Glynn LG, Valderas JM, Healy P, et al. The prevalence of multimorbidity in primary care and its effect on health care utilization and cost. Fam Pract 2011;28:516-23.

17. Wolff JL, Starfield B, Anderson G. Prevalence, expenditures, and complications of multiple chronic conditions in the elderly. Arch Intern Med 2002;162:2269-76.

18. NHS National Services Scotland, Information Services Division. Practice Team Information (PTI) statistics. Edinburgh (UK): The Division; 2010. Available: www.isdscotland.org/HealthTopics/General-Practice/PTI-Statistics/ (accessed 2012 Nov. 14).

19. NHS National Services Scotland, Information Services Division. NHS hospital data quality: towards better data from Scottish hospitals: an assessment of SMRO1 and associated data 2004 2006. Edinburgh (UK): The Division; 2007.

20. Office of the Chief Statistician. Scottish Index of Multiple Deprivation: 2009 general report. Edinburgh (UK): The Scottish Government; 2010.

21. NHS National Services Scotland, Information Services Division. Navigator user guide: potentially preventable admissions indicator. v4.05. Edinburgh (UK): The Division; 2010.

22. Smith SM, Soubhi H, Fortin M, et al. Interventions for improving outcomes in patients with multimorbidity in primary care and community settings. Cochrane Database Syst Rev 2012;(4):CD006560.

23. Ellis G, Whitehead MA, Robinson D, et al. Comprehensive geriatric assessment for older adults admitted to hospital: metaanalysis of randomised controlled trials. BMJ 2011;343:d6553.

24. Wachter RM, Bell D. Renaissance of hospital generalists. BMJ 2012;344:e652.

25. Friedman B, Jiang HJ, Elixhauser A. Costly hospital readmissions and complex chronic illness. Inquiry 2008-2009;45:408-21.

26. Murphy NF, Simpson CR, McAlister FA, et al. National survey of the prevalence, incidence, primary care burden, and treatment of heart failure in Scotland. Heart 2004;90:1129-36.

Affiliations: From the Cambridge Centre for Health Services Research (Payne, Abel), University of Cambridge, Cambridge, UK; Quality, Safety and Informatics Research Group, Population Health Sciences Division (Guthrie), University of Dundee, Dundee, UK; and the Institute of Health and Wellbeing (Mercer), University of Glasgow, Glasgow, UK

Contributors: Rupert Payne, Bruce Guthrie and Stewart Mercer conceived the study. All of the authors were involved in designing the study. Rupert Payne and Gary Abel analyzed the data. Rupert Payne drafted the manuscript. All of the authors interpreted the data, revised the manuscript for important intellectual content and approved the final version submitted for publication. Rupert Payne is the study's guarantor.

Funding: Rupert Payne is supported by a National Institute for Health Research Clinical Lectureship. Gary Abel is supported by a National Institute for Health Research Programme Grant (RP-PG-0608-10050). Stewart Mercer is supported by the Scottish School of Primary Care as national lead for multimorbidity research. Stewart Mercer and Bruce Guthrie are supported by a National Health Service Applied Research Programme Grant from the Chief Scientist Office of the Scottish Government (ARPG/07/01). The funders had no role in the design of the study, the collection, analysis or interpretation of data, the writing of the report or the decision to submit the article for publication.

Acknowledgement: This work was approved by the Privacy Advisory Committee of NHS National Services Scotland. 